\title{
Second and third virial coefficients of low-molecular- weight polystyrene with a benzyl end in toluene
}

\author{
Hiroshi Okada, Shuhei Matsumoto and Yo Nakamura
}

Light-scattering measurements were taken on six samples of polystyrene (PS) with a benzyl group at one end of the chain (benzyl-PS) in toluene at $15^{\circ} \mathrm{C}$. The second and third virial coefficients ( $A_{2}$ and $A_{3}$, respectively) were determined as functions of weight-average molecular weight, $M_{\mathrm{w}}$, which ranges from $6.0 \times 10^{2}$ to $1.2 \times 10^{4}$. It was found that values of $A_{2}$ for benzyl-PS were systematically smaller than those for PS with a butyl group at one end of the chain (butyl-PS) and that the difference becomes larger with decreasing $M_{\mathrm{w}}$. From the $A_{2}$ data obtained, the excess binary-cluster integral between the butyl end and the middle segments was estimated to be about three times larger than the binary-cluster integral between the benzyl end and the middle segments, where 'excess' means the difference from the binary-cluster integral between middle segments. The $A_{3}$ values for benzyl-PS for $M_{\mathrm{w}}<10^{4}$ were also smaller than those for butyl-PS. If we assume a term independent of molecular weight in $A_{3}$, the excess ternary-cluster integral among one butyl end and two middle segments was estimated to be about five times larger than that among one benzyl end and two middle segments.

Polymer Journal (2010) 42, 386-390; doi:10.1038/pj.2010.11; published online 10 March 2010

Keywords: chain-end effect; good solvent; light scattering; polystyrene; second virial coefficient; third virial coefficient

\section{INTRODUCTION}

Solution properties of long flexible polymers are discussed on the basis of the two-parameter theory. ${ }^{1,2}$ However, this theory cannot describe properties in low-molecular-weight regions, in which effects from stiffness and/or local conformations of the chain, chain-end groups and three-segment interactions become essential. ${ }^{3}$

The importance of the effects of chain stiffness on $A_{2}$ was discussed by Yamakawa. ${ }^{4}$ Einaga et al. ${ }^{5}$ later showed that the effects of chain ends, as well as chain stiffness, must be considered to obtain a quantitative agreement between the theoretical and observed values of $A_{2}$ for polystyrene (PS) in toluene (a good solvent) for $M_{\mathrm{w}}<10^{4}$, where $M_{\mathrm{w}}$ denotes the weight-average molecular weight. The PS samples used by Einaga et al. ${ }^{5}$ had a butyl group at one end of the chain. If we perform a similar study on PS samples with different chain ends, different results may be obtained.

It is known that data for $A_{2}$ in cyclohexane solutions of PS with a butyl group (butyl-PS) versus PS with a benzyl group (benzyl-PS) at one end of the chain show very different tendencies for $M_{\mathrm{w}}<10^{4}$ at the theta point $\left(34.5^{\circ} \mathrm{C}\right)$, where $A_{2}$ for sufficiently large $M_{\mathrm{w}}$ vanishes. The positive $A_{2}$ of butyl-PS increasing with decreasing $M_{\mathrm{W}}$ is attributed to effects from the chain ends. ${ }^{4}$ On the other hand, values of $A_{2}$ for benzyl-PS became negative and decreased with decreasing $M_{\mathrm{w}}{ }^{6}$ It was considered that the negative values of $A_{2}$ occurred because of the effects of the three-segment interactions and that effects from chain ends were negligibly small. From these results on the theta solvent system, we may expect to reduce the chain-end effect on $A_{2}$ for a good solvent system by changing the chain end from a butyl group to a benzyl group.

Compared with $A_{2}$, the number of studies on the third virial coefficient, $A_{3}$, of polymer solutions is much smaller because of the difficulty of obtaining precise values. Sato et al. ${ }^{7}$ overcame this problem by applying the Bawn $\operatorname{plot}^{8}$ (see Results and Discussion section), which enables a separate determination of $A_{2}$ and $A_{3}$. Nakamura et al. ${ }^{9}$ applied this plot to PS+benzene solutions to obtain systematic data on $A_{2}$ and $A_{3}$ as functions of $M_{\mathrm{w}}$. They showed that the reduced third virial coefficient, $g$, defined by $A_{3} / A_{2}{ }^{2} M_{\mathrm{w}}$, is an increasing function of $M_{\mathrm{w}}$ for $M_{\mathrm{w}} \gtrsim 10^{5}$. This is consistent with the prediction from the two-parameter theory, except that the values of $g$ were almost constant for $10^{4} \lesssim M_{\mathrm{w}} \lesssim 10^{5}$. Norisuye et al. ${ }^{10}$ examined $g$ in the low-molecular-weight range and found that three-segment interaction has a more important role than chain stiffness. Later, Osa et al. ${ }^{11}$ reached essentially the same conclusion from a study on poly ( $\alpha$-methylstyrene) in toluene. In these studies, only data for $M_{\mathrm{w}}>10^{4}$ were discussed without considering the effects from chain ends. Because the effect of the chain end on $A_{3}$ of butyl-PS in cyclohexane is known to be remarkable for $M_{\mathrm{w}} \lesssim 10^{4}, 12$ the same effect on $A_{3}$ should be considered for good solvent systems.

In this study, we performed light-scattering measurements to determine $A_{2}$ and $A_{3}$ for benzyl-PS, with $M_{\mathrm{w}}$ ranging from $6.0 \times 10^{2}$ to $1.2 \times 10^{4}$ in toluene at $15^{\circ} \mathrm{C}$. This enabled us to study the effects from the chain end and chain stiffness by comparing the results with the data for butyl-PS. ${ }^{5}$ 


\section{EXPERIMENTAL PROCEDURE}

Benzyl-PS samples (PS600, PS700, PS4800, PS8300 and PS12000) were obtained by anionic polymerization of styrene with benzyllithium as the initiator ${ }^{13}$ in an argon atmosphere. These samples, except the two lowest molecular-weight samples, were fractionally precipitated with benzene as the solvent and methanol as the precipitant. Sample PS1900 was prepared in the previous study. ${ }^{6}$ The number-average to weight-average molecular weight ratio, $M_{\mathrm{w}} / M_{\mathrm{n}}$, was determined by gel-permeation chromatography. Values were less than 1.06, as shown in Table 1. The two samples, PS600 and PS700, were obtained by fractionation of low-molecular-weight benzyl-PS by gel-permeation chromatography with chloroform as the eluent, and they were then reprecipitated from acetone solutions to water. These liquid samples were dried at $50^{\circ} \mathrm{C}$ in a rotary evaporator for 2 weeks. Existence of the benzyl chain end was confirmed by ${ }^{1} \mathrm{H}-\mathrm{NMR}$ and MALDI-TOF mass spectroscopy.

Each test solution was optically clarified by filtration through a double layer of Teflon filters, with $0.1 \mu \mathrm{m}$ pores, into a cylindrical light-scattering cell. The cell had been rinsed with refluxing acetone for more than $1.5 \mathrm{~h}$ and dried overnight in a desiccator.

The polymer mass concentration, $c$, of each solution was calculated from the gravimetrically determined polymer weight fraction, $w$, multiplied by the solution density $\rho$, which was calculated from $\rho=\rho_{0}\left[1+\left(1-\bar{v} \rho_{0}\right) w\right]$. Here, $\rho_{0}$ and $\bar{v}$ denote the solvent density and the specific volume of the solute, respectively. The specific volumes for PS600 and PS700 were determined from density measurements of the solvent and the solutions by an Anton-Paar densitometer, DMA-5000 (Anton-Paar, Graz, Austria), as shown in Table 1. The value for the other samples was assumed to be $0.91 \mathrm{~cm}^{3} \mathrm{~g}^{-1} .{ }^{14}$

Light-scattering measurements were taken on a Fica 50 light-scattering photometer (Fica, Saint Denis, France) with vertically polarized light with a wavelength of $436 \mathrm{~nm}$ in an angular range from 22.5 to $142.5^{\circ}$ to obtain the excess Rayleigh ratio, $R_{\theta}$, at the scattering angle $\theta$. The data for each solution were extrapolated to $\theta=0$ by plotting $K c / R_{\theta}$ against $\sin ^{2} \theta$ to obtain the zeroangle value $K c / R_{0}$, where $K$ represents the optical constant. Corrections of optical anisotropy were made for both solutions and solvent according to the following equation:

$$
R_{0}=\left(1-\frac{7}{6} \rho_{u}\right) R_{0}^{*}
$$

Here, $R_{0}{ }^{*}$ denotes the uncorrected $R_{0}$ and $\rho_{u}$ is the depolarized ratio, which was determined by the method of Rubingh and Yu. ${ }^{15}$

The specific reflective index increments, $\partial n / \partial c$, measured by a modified Schulz-Cantow-type differential refractometer, are summarized in the fifth column of Table 1.

\section{RESULTS AND DISCUSSION}

Data analysis

The scattering intensities, extrapolated to $\theta=0$, were obtained as a function of $c$, for each polymer sample. Except PS600 and PS700, they were analyzed using the Bawn plot, ${ }^{8}$ that is, $S\left(c_{1}, c_{2}\right)$, as defined by the following equation plotted against $c_{1}+c_{2}:{ }^{7}$

$$
\begin{aligned}
S\left(c_{1}, c_{2}\right) & \equiv \frac{\left(K c / R_{0}\right)_{c=c_{2}}-\left(K c / R_{0}\right)_{c=c_{1}}}{c_{2}-c_{1}} \\
& =2 A_{2}+3 A_{3}\left(c_{1}+c_{2}\right)+\cdots
\end{aligned}
$$

Here, $\left(K c / R_{0}\right)_{c=c_{i}}$ means $\left(K c / R_{0}\right)$ at $c=c_{i}(i=1,2)$. Figure 1 shows the Bawn plots for the samples indicated. Each plot can be fitted to a straight line. The slope and intercept at $c_{1}+c_{2}=0$ are equated to $3 A_{3}$ and $2 A_{2}$, respectively, according to Equation (1). We tried to apply the same plot to the data for PS600 and PS700, but we could not draw a line uniquely for each plot because of the considerable scattering of data points. For these samples, we applied the Berry plot, ${ }^{16}$ that is, $\left(K c / R_{0}\right)^{1 / 2}$ against $c$, as is shown in Figure 2. The slope and the intercept at $c=0$ of the fitted straight line for each plot are equated to $M_{\mathrm{w}}{ }^{1 / 2} A_{2}$ and $M_{\mathrm{w}}{ }^{-1 / 2}$, respectively. The values for $A_{2}$ and $A_{3}$, along with $M_{\mathrm{w}}$, are summarized in Table 1 .

\section{Second virial coefficient}

The dependence of $A_{2}$ on molecular weight is illustrated in Figure 3. The unfilled and filled circles represent the data points of $A_{2}$ for benzyl- and butyl-PS, ${ }^{5}$ respectively, in toluene at $15^{\circ} \mathrm{C}$. The data points for benzyl-PS are systematically lower than those for butyl-PS. The difference becomes larger with decreasing $M_{\mathrm{w}}$.

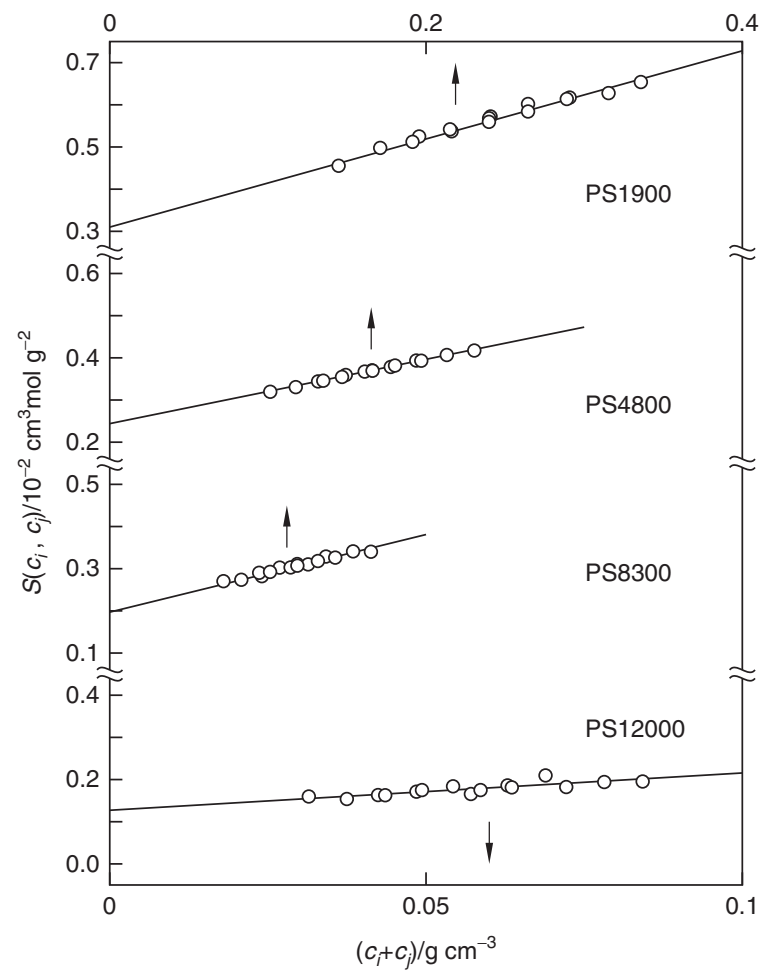

\begin{tabular}{|c|c|c|c|c|c|c|}
\hline Sample & $10^{-2} M_{w}$ & $M_{w} / M_{n}$ & $\overline{\mathrm{V}}\left(\mathrm{cm} \mathrm{m}^{3} \mathrm{~g}^{-1}\right)$ & $\partial n / \partial c\left(c m^{3} g^{-1}\right)$ & $10^{4} \mathrm{~A}_{2}\left(\mathrm{~cm}^{3} \mathrm{molg}^{-2}\right)$ & $10^{3} A_{3}\left(\mathrm{~cm}^{6} \mathrm{molg}^{-3}\right)$ \\
\hline PS600 & 6.25 & 1.02 & 0.939 & $0.099_{2}$ & 25.0 & - \\
\hline PS700 & 6.94 & 1.02 & 0.935 & $0.102_{3}$ & 21.6 & - \\
\hline PS1900 & 19.8 & 1.06 & - & $0.106_{0}$ & 15.5 & 3.5 \\
\hline PS4800 & 47.9 & 1.05 & - & $0.108_{0}$ & 12.2 & 2.5 \\
\hline PS8300 & 83.2 & 1.04 & - & $0.109_{8}$ & $9.8_{5}$ & 2.9 \\
\hline PS12000 & 116 & 1.03 & - & $0.110_{3}$ & $8.8_{6}$ & 3.0 \\
\hline
\end{tabular}

Figure 1 Bawn plots for benzyl-end polystyrene samples in toluene at $15^{\circ} \mathrm{C}$.

Table 1 Properties of benzyl-end polystyrene samples in toluene at $15^{\circ} \mathrm{C}$ 


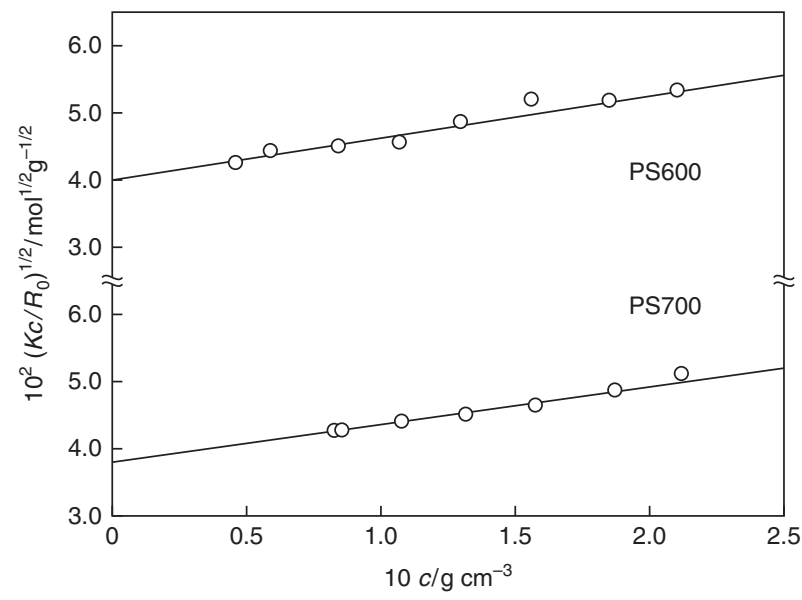

Figure 2 Square-root plots for benzyl-end polystyrene samples in toluene at $15^{\circ} \mathrm{C}$.

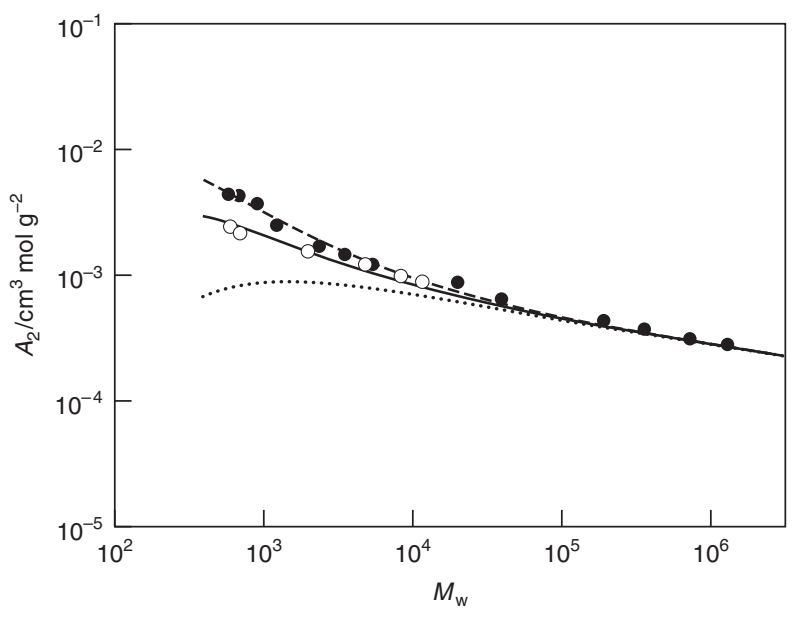

Figure 3 Dependence of $A_{2}$ on molecular weight for polystyrenes in toluene at $15.0^{\circ} \mathrm{C}$. Filled and unfilled circles represent data for butyl-end ${ }^{5}$ and benzyl-end polystyrenes, respectively. The dotted curve indicates theoretical values without chain-end effects. Solid and dashed curves represent theoretical values with chain-end effects.

Theoretically, $A_{2}$ is expressed by the following equation: ${ }^{4}$

$$
A_{2}=A_{2}^{(\mathrm{HW})}+A_{2}^{(\mathrm{E})}
$$

$A_{2}{ }^{(\mathrm{HW})}$ represents the term for the binary interactions between chain segments, not including the contribution of the chain-end effects, and may be calculated from the following equation for the helical wormlike (HW) chain ${ }^{3}$ with beads or segments arrayed along the chain contour with a distance of $a$ :

$$
A_{2}^{(\mathrm{HW})}=\frac{N_{\mathrm{A}} c_{\infty}^{3 / 2} L^{2} B}{2 M^{2}} h
$$

$N_{\mathrm{A}}, L$ and $M$ denote the Avogadro constant, the contour length and molecular weight, respectively. $c_{\infty}$ is expressed $a^{3}$

$$
c_{\infty}=\frac{4+\left(\lambda^{-1} \tau_{0}\right)^{2}}{4+\left(\lambda^{-1} \kappa_{0}\right)^{2}+\left(\lambda^{-1} \tau_{0}\right)^{2}}
$$

with the stiffness parameter, $\lambda^{-1}$, and the differential geometrical curvature, $\kappa_{0}$, and torsion, $\tau_{0}$. The excluded-volume parameter, $B$, is defined by

$$
B=\frac{\beta_{2}}{a^{2} c_{\infty}^{3 / 2}}
$$

$\beta_{2}$ is the binary-cluster integral between middle segments. Here, the word middle means the segments excluding the ones at the chain ends. The dimensionless $h$ in Equation (3) may be calculated from the modified Barrett equation ${ }^{4,17}$ as a function of $\lambda B$ and $\lambda L$ with the scaled excluded-volume parameter (see Einaga et al. ${ }^{5}$ ).

The chain-end term, $A_{2}{ }^{(\mathrm{E})}$, may be expressed as ${ }^{4}$

$$
A_{2}^{(\mathrm{E})}=a_{2,1} M^{-1}+a_{2,2} M^{-2}
$$

The constants $a_{2,1}$ and $a_{2,2}$ are defined as ${ }^{4}$

$$
\begin{aligned}
& a_{2,1}=2 N_{A} \beta_{2,1} / M_{0} \\
& a_{2,2}=2 N_{A} \Delta \beta_{2,2}
\end{aligned}
$$

with

$$
\begin{aligned}
& \beta_{2,1}=\left(\beta_{01}+\beta_{02}\right) / 2 \\
& \Delta \beta_{2,2}=\left(\beta_{11}+2 \beta_{12}+\beta_{22}\right) / 4-2 \beta_{2,1}
\end{aligned}
$$

$M_{0}$ is the molecular weight for one segment. $\beta_{i j}$ is the excess binarycluster integral between segments $i$ and $j$ over the binary-cluster integral between the middle segments, $\beta_{2}$, where the subscripts 0,1 and 2 represent the segments at the middle, at one of the chain ends and at the other chain end, respectively. A monomer unit is regarded as a segment.

The dotted line in Figure 3 represents the calculated $A_{2}{ }^{(\mathrm{HW})}$ from Equation (3), with the following parameter set for PS in toluene: ${ }^{18}$ $M_{\mathrm{L}}=358 \mathrm{~nm}^{-1}, \lambda^{-1}=2.06 \mathrm{~nm}, \lambda^{-1} \kappa_{0}=3.0, \lambda^{-1} \tau_{0}=6.0$ and $\lambda B=0.33$. $M_{\mathrm{L}}$ is the molecular weight for the unit contour length. The calculated values for $M_{\mathrm{w}} \lesssim 10^{5}$ are systematically lower than both experimental values for butyl- and benzyl-PS. The difference between theoretical and experimental values becomes larger with decreasing $M_{\mathrm{w}}$. By adding $A_{2}{ }^{(E)}$ with $a_{2,1}=2.5 \mathrm{~cm}^{3} \mathrm{~g}^{-1}$ and $a_{2,2}=-200 \mathrm{~cm}^{3} \mathrm{~mol}^{-1,5}$ we obtain the dashed line, which closely fits the experimental data for butyl-PS. The solid line calculated with $a_{2,1}=1.4 \mathrm{~cm}^{3} \mathrm{~g}^{-1}$ and $a_{2,2}=-200 \mathrm{~cm}^{3} \mathrm{~mol}^{-1}$ also closely fits the data for benzyl-PS. From Equation (7), with these values of $a_{2,1}$, we calculate $\beta_{2,1}$ for butyl- and benzyl-PS to be 0.22 and $0.12 \mathrm{~nm}^{3}$, respectively.

Both ends of the benzyl-PS chain have the same chemical structure, because a polystyryl anion gives the benzyl structure when it is deactivated with a proton. Thus, we may regard $\beta_{01}=\beta_{02}$ in Equation (9) for benzyl-PS to obtain $\beta_{01}=0.12 \mathrm{~nm}^{3}$ for the binary interaction between the benzyl end and middle (or PS) segments. The structure of the terminated end of butyl-PS is the same as that of benzyl-PS. As the values for $\beta_{01}$ for both polymers are the same, we obtain $0.32 \mathrm{~nm}^{3}$ for $\beta_{02}$ from Equation (9) with the above $\beta_{01}$ and $\beta_{2,1}$ for butyl-PS. The value of $\beta_{02}$, which represents the binary interaction between the butyl end and middle segments, is about three times larger than $\beta_{01}$ for the benzyl end.

Positive $\beta_{01}$ shows that the net binary-cluster integral between the benzyl end and middle segments is larger than the one between middle segments, $\beta_{2}$. These results are rather different from those for PS in cyclohexane $\left(34.5^{\circ} \mathrm{C}\right)$, a theta solvent, in which $\beta_{01}$ is vanishingly small. ${ }^{6}$ In this solvent, the binary-cluster integral between the benzyl end and middle (PS) segments may be incidentally close to the one between middle segments.

\section{Third virial coefficient}

Figure 4 shows the dependence of $A_{3}$ on molecular weight, in which literature data for butyl-PS in toluene at $15^{\circ} \mathrm{C}^{19}$ and those 


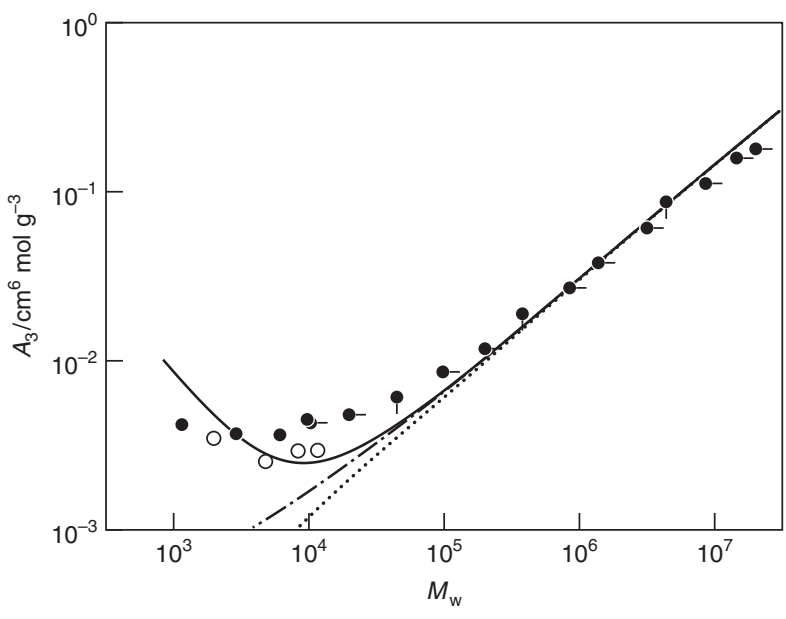

Figure 4 Dependence of $A_{3}$ on molecular weight for polystyrenes in good solvents. Filled and unfilled circles represent data for butyl-end (no pip, in toluene at $15^{\circ} \mathrm{C} ;{ }^{19}$ pip down ${ }^{7}$ and right, ${ }^{9}$ in benzene at $25^{\circ} \mathrm{C}$ ) and benzylend polystyrenes, respectively. Dotted, dot-dashed and solid curves represent theoretical values for $A_{3, \beta_{2}}^{(\mathrm{HW})}, A_{3, \beta_{2}}^{(\mathrm{HW})}+A_{3, \beta_{3}}^{(\mathrm{O})}$, and $A_{3, \beta_{2}}^{(\mathrm{HW})}+A_{3, \beta_{3}}^{(\mathrm{O})}+A_{3, \beta_{3}}^{(\mathrm{E})}$, respectively (with $\beta_{3}=4.7 \times 10^{-3} \mathrm{~nm}^{6}$ ).

for butyl-PS in benzene at $25^{\circ} \mathrm{C}^{7,9}$ are included. The values of $A_{3}$ for benzyl-PS from this study are slightly lower than those for butyl-PS.

The third virial coefficient for the linear chain may be expressed as ${ }^{12}$

$$
A_{3}=A_{3, \beta_{2}}^{(\mathrm{HW})}+A_{3, \beta_{3}}
$$

The first and second terms on the right-hand side of the above equation correspond to the contribution of the binary- and ternarysegment interactions, respectively. We note that the remaining part, which includes cross terms of $\beta_{2}$ and $\beta_{3},{ }^{20}$ is ignored here because it is anticipated to be negligibly small.

The first term on the right-hand side of Equation (11) may be expressed as

$$
A_{3, \beta_{2}}^{(\mathrm{HW})}=g_{2}\left[A_{2}^{(\mathrm{HW})}\right]^{2} M
$$

$g_{2}$ may be calculated from the following equation: ${ }^{10,11}$

$$
g_{2}=2.219 \bar{z}\left(1+18 \bar{z}+12.6 \bar{z}^{2}\right)^{-1 / 2}
$$

$\bar{z}$ is defined as

$$
\bar{z}=\frac{z}{\alpha_{S}^{3}}
$$

with

$$
z=\left(\frac{3}{2 \pi}\right)^{3 / 2}(\lambda B)(\lambda L)^{1 / 2}
$$

The radius expansion factor, $\alpha_{S}$, may be calculated from the modified Domb-Barrett equation: ${ }^{21}$

$$
\begin{aligned}
\alpha_{S}^{2}= & {\left[1+10 \tilde{z}+(70 \pi / 9+10 / 3) \tilde{z}^{2}+8 \pi^{3 / 2} \tilde{z}^{3}\right]^{2 / 15} } \\
& \times\left[0.933+0.067 \exp \left(-0.85 \tilde{z}-1.39 \tilde{z}^{2}\right)\right]
\end{aligned}
$$

with

$$
\tilde{z}=\frac{3}{4} K(\lambda L) z
$$

where $K(\lambda L)$ is a known function. ${ }^{3,22}$

The second term on the right-hand side of Equation (11) may be expressed as ${ }^{12}$

$$
A_{3, \beta_{3}}=A_{3, \beta_{3}}^{(0)}+A_{3, \beta_{3}}^{(\mathrm{E})}
$$

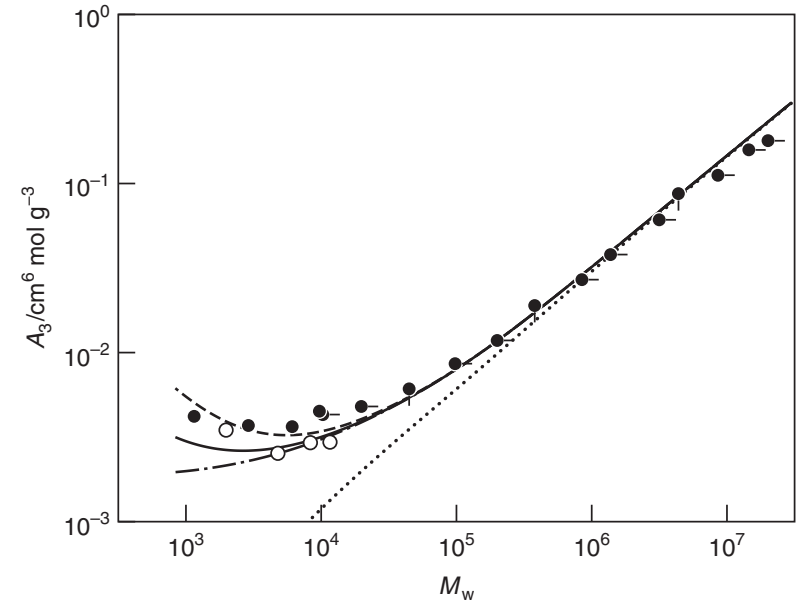

Figure 5 Dependence of $A_{3}$ on molecular weight for polystyrenes in good solvents. Symbols and dotted and dashed curves have the same meaning as those in Figure 4. Solid and dashed lines represent theoretical values for benzyl- and butyl-end polystyrenes, respectively (with $\beta_{3}=1.8 \times 10^{-2} \mathrm{~nm}^{6}$ ).

where the first term on the right-hand side represents the contribution from the interaction among three middle segments. The other term is for the interaction among two middle segments and one chain-end segment.

The zeroth-order contribution to the former term can be expressed as ${ }^{1,23}$

$$
A_{3, \beta_{3}}^{(0)}=\frac{N_{A}^{2} n^{3} \beta_{3}}{3 M^{3}}
$$

with the ternary-cluster integral, $\beta_{3}$, among middle segments. If we ignore the higher term of the order of $M^{-2}$, we may express the chain-end term as ${ }^{12}$

$$
A_{3, \beta_{3}}^{(\mathrm{E})}=a_{3,1} M^{-1}
$$

Here, $a_{3,1}$ is given by

$$
a_{3,1}=2 N_{A}^{2} \beta_{3,1} / M_{0}^{2}
$$

with

$$
\beta_{3,1}=\left(\beta_{001}+\beta_{002}\right) / 2
$$

$\beta_{00 i}$ denotes the excess ternary-cluster integrals among two middle segments (denoted by 0 ) and one end segment $i(i=1,2)$ over that among three middle segments $\beta_{3}$.

The dotted and dot-dashed lines in Figure 4 show $A_{3, \beta_{2}}^{(\mathrm{HW})}$ and $A_{3, \beta_{2}}^{(\mathrm{HW})}+A_{3, \beta_{3}}^{(0)}$, respectively, according to the above equations, with the parameter set for PS in toluene given in the previous section. Here, we take $\beta_{3}=4.7 \times 10^{-3} \mathrm{~nm}^{6}$, which was obtained from the value of $A_{3}$ of sufficiently high-molecular-weight PS in cyclohexane at the theta point. ${ }^{24}$ Both lines cannot explain the experimental data for $M_{\mathrm{w}} \lesssim 10^{5}$. By adding $A_{3, \beta_{3}}^{(\mathrm{E})}$ with $a_{3,1}=8 \mathrm{~cm}^{6} \mathrm{~g}^{-2}$ to the dot-dashed line, we obtain the solid line, which is somewhat closer to the data points. However, the agreement is limited to a restricted molecular weight range.

A better fit can be obtained if we use $\beta_{3}=1.8 \times 10^{-2} \mathrm{~nm}^{6}$, a much larger $\beta_{3}$ than the one used above, as shown by the dot-dashed line in Figure 5 for $A_{3, \beta_{2}}^{(\mathrm{HW})}+A_{3, \beta_{3}}^{(0)}$. Use of such a large $\beta_{3}$ was suggested by Koyama and Sato. ${ }^{19}$ The solid and dashed lines in Figure 5 were obtained by adding $A_{3, \beta_{3}}^{(\mathrm{E})}$ with $a_{3,1}=1.0$ and $3.0 \mathrm{~cm}^{6} \mathrm{~g}^{-2}$, respectively, to the dot-dashed line. This led to satisfactory fits to the data points for benzyl- and butyl-PS, respectively. From these values of $a_{3,1}$, we calculate $\beta_{3,1}$ to be 0.015 and $0.045 \mathrm{~nm}^{6}$ 
for the benzyl- and butyl-PS, respectively. The former $\beta_{3,1}$ gives $\beta_{001}=0.015 \mathrm{~nm}^{6}$ for the benzyl end. The latter, with this $\beta_{001}$, gives $\beta_{002}=0.075 \mathrm{~nm}^{6}$ for the butyl end. These results show that $\beta_{002}$ is about five times larger than $\beta_{001}$.

The $\beta_{3}$ value used for Figure 5 suggests that $\beta_{3}$ in toluene at $15^{\circ} \mathrm{C}$ is about four times larger than that in cyclohexane at $34.5^{\circ} \mathrm{C}$. This seems rather odd because calculations on rigid spheres with the LennardJones potential show that $\beta_{3}$ at the Boyle point (corresponding to the theta point) is larger than the one at higher temperatures. ${ }^{25}$ Furthermore, if we calculate $\beta_{3} / \beta_{2}{ }^{2}$ from the above $\beta_{3}$ and $\beta_{2}=0.041 \mathrm{~nm}^{3}$, as estimated from $\lambda B=0.33$, we obtain $\beta_{3} / \beta_{2}{ }^{2}=11$, which is much larger than the rigid-sphere value of 0.625 . However, the $\beta_{3}$ term of Equation (19) only adds a constant to $A_{3}$. It is likely that we overlooked a contribution independent of molecular weight, although we do not know what it is.

\section{CONCLUSION}

By combining the data for $A_{2}$ and $A_{3}$ for low-molecular-weight butyl- and benzyl-PS, we estimated the excess binary-cluster integrals $\left(\beta_{01}\right.$ and $\left.\beta_{02}\right)$ between chain-end and middle segments, as well as the excess ternary-cluster integrals $\left(\beta_{001}\right.$ and $\left.\beta_{002}\right)$ among one chain-end segment and two middle segments. The conclusions obtained from the study are summarized as follows: (1) $\beta_{02}$ for the interaction between the butyl end and middle segments is about three times larger than $\beta_{01}$ for the interaction between the benzyl end and middle segments. (2) The data analysis of $A_{3}$ suggested a contribution that is independent of molecular weight by some effect. (3) If we assume such a contribution, $\beta_{002}$ for the interaction among one butyl end and two middle segments is estimated to be about five times larger than $\beta_{001}$ for the interaction among one benzyl end and two middle segments.

\section{ACKNOWLEDGEMENTS}

We thank Professor Takenao Yoshizaki of Kyoto University for valuable discussions.

1 Yamakawa, H. Modern Theory of Polymer Solutions (Harper \& Row, New York, 1971). 2 Fujita, H. Polymer Solutions (Elsevier, Amsterdam, 1990).
3 Yamakawa, H. Helical Wormlike Chains in Polymer Solutions (Springer, Berlin, 1997). 4 Yamakawa, H. On the theory of the second virial coefficient for polymer chains. Macromolecules 25, 1912-1916 (1992).

5 Einaga, Y., Abe, F. \& Yamakawa, H. Second virial coefficients of oligo- and polystyrenes. Effects of chain ends. Macromolecules 26, 6243-6250 (1993).

6 Mizuno, T., Terao, K., Nakamura, Y. \& Norisuye, T. Second and third virial coefficients of polystyrene with benzyl ends near the $\Theta$ point. Macromolecules 38, 4432-4437 (2005).

7 Sato, T., Norisuye, T. \& Fujita, H. Second and third virial coefficients for binary polystyrene mixtures in benzene. J. Polym. Sci. Part B: Polym. Phys. 25, 1-17 (1987).

8 Bawn, C. E. H., Freeman, R. F. J. \& Kamaliddin, A. R. High polymer solutions part II. The osmotic pressures of polystyrene solutions. Trans. Faraday Soc. 46, 862-872 (1950).

9 Nakamura, Y., Norisuye, T. \& Teramoto, A. Third virial coefficient of polystyrene in benzene. J. Polym. Sci. Part B: Polym. Phys. 29, 153-159 (1991).

10 Norisuye, T., Nakamura, Y. \& Akasaka, K. Reduced third virial coefficient for linear flexible polymers in good solvents. Macromolecules 26, 3791-3794 (1993).

11 Osa, M., Yoshizaki, T. \& Yamakawa, H. Some comments on the analysis of the third virial coefficient for polymer chains. Polym. J. 36, 634-641 (2004).

12 Yamakawa, H., Abe, F. \& Einaga, Y. Effects of chain ends on the third virial coefficient for polymer chains. Oligo- and polystyrenes and oligo- and poly(methyl methacrylate)s. Macromolecules 27, 3272-3275 (1994).

13 Tsukahara, Y., Inoue, J., Ohta, Y., Kohjiya, S. \& Okamoto, Y. Preparation and characterization of $\alpha$-benzyl- $\omega$-vinylbenzyl polystyrene macromonomer. Polym. J. 26, 1013-1018 (1994).

14 Lechner, M. D. \& Steinmeier, D. G. Polymer Handbook, 3rd Ed. (eds. Brandrup, J. \& Immergut, E. H.), Ch. VII, 61-148 (Wiley Interscience, 1989).

15 Rubingh, D. N. \& Yu, H. Characterization of stiff chain macromolecules. Poly( $n$-hexyl isocyanate) in $n$-hexane. Macromolecules 9, 681-685 (1976).

16 Berry, G. C. Thermodynamic and conformational properties of polystyrene. I. Lightscattering studies on dilute solutions of linear polystyrenes. J. Chem. Phys. 44, 45504564 (1966).

17 Barrett, A. J. Second osmotic virial coefficient for linear excluded volume polymer solutions. Macromolecules 18, 196-200 (1985).

18 Abe, F., Einaga, Y., Yoshizaki, T. \& Yamakawa, H. Excluded-volume effects on the meansquare radius of gyration of oligo- and polystyrenes in dilute solutions. Macromolecules 26, 1884-1890 (1993).

19 Koyama, R. \& Sato, T. Thermodynamic properties of toluene solutions of low molecular weight polystyrenes over wide ranges of concentration. Macromolecules 35, 2235-2242 (2002).

20 Norisuye, T. \& Nakamura, Y. Effects of three-segment interactions on the temperature dependence of the third viral coefficient for flexible chains near the $\Theta$ point. Macromolecules 27, 2054-2057 (1994).

21 Domb, C. \& Barrett, A. J. Universality approach to the expansion factor of a polymer chain. Polymer 17, 179-184 (1976).

22 Shimada, J. \& Yamakawa, H. Statistical mechanics of helical worm-like chains. XV. Excluded-volume effects. J. Chem. Phys. 85, 591-600 (1986).

$23 \mathrm{Zimm}, \mathrm{B}$. H. Application of the methods of molecular distribution to solutions of large molecules. J. Chem. Phys. 14, 164-179 (1946).

24 Nakamura, Y., Norisuye, T. \& Teramoto, A. Second and third virial coefficients for polystyrene in cyclohexane near the $\Theta$ point. Macromolecules 24, 4904-4908 (1991).

25 Barker, J. A., Leonard, P. J. \& Pompe, A. Fifth virial coefficients. J. Chem. Phys. 44, 4206-4211 (1966). 